\title{
Multivariate Heteroscedasticity Models for Functional Brain Connectivity
}

\author{
Christof Seiler* and Susan Holmes \\ Department of Statistics, Stanford University, Stanford, CA, United States
}

Functional brain connectivity is the co-occurrence of brain activity in different areas during resting and while doing tasks. The data of interest are multivariate timeseries measured simultaneously across brain parcels using resting-state fMRI (rfMRI). We analyze functional connectivity using two heteroscedasticity models. Our first model is low-dimensional and scales linearly in the number of brain parcels. Our second model scales quadratically. We apply both models to data from the Human Connectome Project (HCP) comparing connectivity between short and conventional sleepers. We find stronger functional connectivity in short than conventional sleepers in brain areas consistent with previous findings. This might be due to subjects falling asleep in the scanner. Consequently, we recommend the inclusion of average sleep duration as a covariate to remove unwanted variation in rfMRI studies. A power analysis using the HCP data shows that a sample size of 40 detects $50 \%$ of the connectivity at a false discovery rate of $20 \%$. We provide implementations using $R$ and the probabilistic programming language Stan.

Keywords: Bayesian analysis, functional connectivity, heteroscedasticity, covariance regression, sleep duration

\section{INTRODUCTION}

Functional connectivity focuses on the exploration of neurophysiological measures of brain activity between brain regions (Friston, 2011; Smith, 2012; Varoquaux and Craddock, 2013). Functional connectivity studies have increased our understanding of the basic structure of the brain (Eguíluz et al., 2005; Sporns et al., 2004; Bassett and Bullmore, 2006; Fox and Raichle, 2007; Bullmore and Sporns, 2009; Van Den Heuvel and Pol, 2010) and provided insights into pathologies (Greicius et al., 2003; Greicius, 2008; Biswal et al., 2010; Fox and Greicius, 2010).

From a statistical viewpoint, functional connectivity is the problem of estimating covariance matrices, precision matrices, or correlation matrices from timeseries data. These matrices encode the level of connectivity between any two brain regions. The timeseries are derived from restingstate fMRI (rfMRI) by averaging individual voxels over parcels in the gray matter. We define parcels manually or with data-driven brain parcellation algorithms. The final goal can be an exploratory or a differential analysis comparing connectivity across regions between experimental conditions and time (Preti et al., 2016). Many statistical methods are available to estimate covariance matrices, precision matrices, or correlation matrices from multivariate data. The sample covariance and its inverse, or the sample correlation matrix are usually poor estimators because of the highdimensionality of the data (large number of parcels $p$ and small number of subjects). The number of parameters grows quadratically in the number of regions with $p(p-1) / 2$ possible pairwise connections between parcels. Therefore more elaborate estimators need to be employed, such as 
the Graphical Lasso (Friedman et al., 2008) estimator for inversecovariance matrices or the Ledoit-Wolf shrinkage estimator (Ledoit and Wolf, 2004) for correlation matrices. Application of these methods to rfMRI are available (Varoquaux et al., 2010a,b; Smith et al., 2011; Ryali et al., 2012; Varoquaux et al., 2012; Liang et al., 2016).

The estimation of connectivity is usually only the first step and leads to downstream differential analyses comparing connectivity between experimental conditions or between subgroups. For instance, we will compare the connectivity of short sleepers with conventional sleepers available as preprocessed timeseries from the Human Connectome Project (Van Essen et al., 2013). One approach is massive univariate testing of each of the $p(p-1) / 2$ connections by linear modeling. Such an approach allows us to test different contrasts and include batch factors or random effect terms (Lewis et al., 2009; Grillon et al., 2013). It lacks statistical power because it ignores possible dependencies between elements in the connectivity matrix. An alternative is to assess selected functionals or summary statistics rather than individual elements in the connectivity matrix (Stam, 2004; Salvador et al., 2005; Achard et al., 2006; Marrelec et al., 2008; Bullmore and Sporns, 2009; Ginestet et al., to appear). Another approach is to flip response variable and explanatory variable and predict experimental condition (or subgroup) from connectivity matrices (or functionals of matrices) through machine learning (Dosenbach et al., 2010; Craddock et al., 2012). These approaches lack interpretability in terms of brain function.

The problem boils down to modeling heteroscedasticity. Heteroscedasticity is said to occur when the variance of the unobservable error, conditional on explanatory variables, is not constant. For example, consider the regression problem predicting expenditure on meals from income. People with higher income will have greater variability in their choices of food consumption. A poorer person will have less choice, and be constrained to inexpensive foods. In functional connectivity, heteroscedasticity is multivariate and variances become covariance matrices. In other words, heteroscedasticity cooccurs among brain parcels and can be explained as a function of explanatory variables.

In this article, we propose a low-dimensional multivariate heteroscedasticity model for functional connectivity. Our model is of intermediary complexity, between modeling all $p(p-1) / 2$ connections and only using global summary statistics. Our model builds on the covariance regression model introduced by Hoff and Niu (2012). It includes a random effects term that describes heteroscedasticity in the multivariate response variable. We adapt it for functional connectivity and implement it using the statistical programming language Stan. Additionally, we perform preliminary thinning of the observed multivariate timeseries from $N$ to the effective sample size $n$. Using $n$ reduces false positives and speeds up computations by a factor of $N / n$. To find the appropriate $n$, we compute the autocorrelation as it is common in the Markov chain Monte Carlo literature. We compare our low-dimensional model to a full covariance model contained in the class of linear covariance models introduced by Anderson (1973). Both models are used to analyze real data from
HCP comparing connectivity between short and conventional sleepers.

From a neuroscience viewpoint, our low-dimensional model is applicable if we belief that multiple brain parcels work together to accomplish cognitive tasks. Even if this assumption is not entirely true, our low-dimensional model can serve a way to simplify functional connectivity analyses and improve interpretability. One can think of a low-dimensional model as a way to reduce the dimensions of the original data to an interpretable number of variables.

\section{MATERIALS AND METHODS}

\subsection{Data}

We analyzed data from the WU-Minn HCP 1200 Subjects Data Release. We focus on the functional-resting fMRI (rfMRI) data of 820 subjects. The images were acquired in four runs of approximately $15 \mathrm{~min}$ each. Acquisition ranged over 13 periods (Q01, Q02, ..., Q13). We separated the subjects into two groups: short sleepers $(\leq 6 \mathrm{~h})$ or conventional sleepers (7-9 h) as defined by the National Sleep Foundation (Hirshkowitz et al., 2015). This results in 489 conventional and 241 short sleepers. The HCP 1200 data repository contains images processed at different levels: spatially registered images, functional timeseries, and connectivity matrices. We work with the preprocessed timeseries data. In particular, the rfMRI preprocessing pipeline includes both spatial (Glasser et al., 2013) and temporal preprocessing (Smith et al., 2013). The spatial preprocessing uses tools from FSL (Jenkinson et al., 2012) and FreeSurfer (Fischl et al., 1999) to minimize distortions and align subject-specific brain anatomy to reference atlases using volume-based and surface-based registration methods. After spatial preprocessing, artifacts are removed from each subject individually (Griffanti et al., 2014; Salimi-Khorshidi et al., 2014), then the data are temporally demeaned and variance stabilized (Beckmann and Smith, 2004), and further denoised using a group-PCA (Smith et al., 2014). Components of a spatial groupICA (Hyvärinen, 1999; Beckmann and Smith, 2004) are mapped to each subject defining parcels (Glasser et al., 2013). The ICA-weighted voxelwise rfMRI signal are averaged over each component. Each weighted average represents one row in the multivariate timeseries. Note that parcels obtained in this way are not necessary spatially contiguous, in particular, they can overlap and include multiple spatially separated regions. HCP provides a range of ICA components 15, 25, 50, 100, 200, and 300. We choose 15 (Figure 1) for our analysis to allow for comparison with prior sleep related findings on a partially overlapping dataset (Curtis et al., 2016).

\subsection{Low-Dimensional Covariance Regression}

In this section, we introduce a low-dimensional linear model to compare connectivity between experimental conditions or subgroups. 

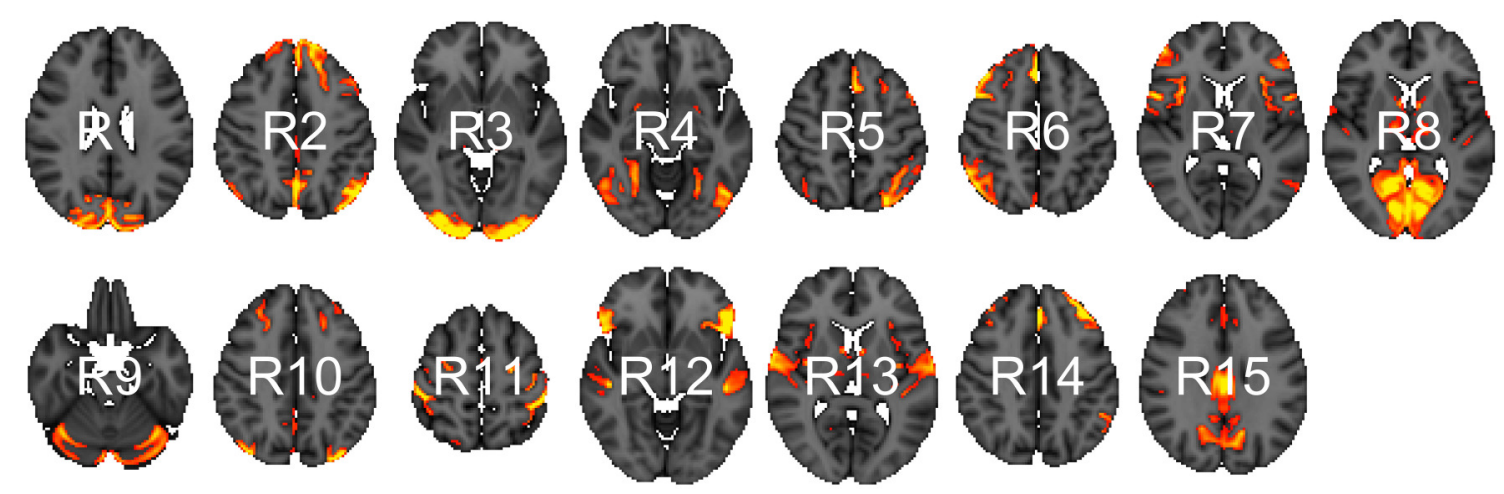

FIGURE 1 | Parcels derived from spatial group-ICA. Created at the most relevant axial slices in MNI152 space. According to Smith et al. (2009), these parcels map to visual areas (R1, R3, R4, and R8), sensorimotor (R7 and R11), cognition-language (R2, R5, R10, and R14), perception-somesthesis-pain (R2, R6, R10, and R14), cerebellum (R9), executive control (R12), auditory (R12 and R13), and default network (R15).

\subsubsection{Model}

The data we observe are $p$-dimensional multivariate vectors $y_{1}, \ldots, y_{N}$. We assume that the observations are mean-centered so that $\frac{1}{N} \sum_{i=1}^{N} y_{i}=0$. After centering, we subsample each timeseries at $n<N$ time points to remove temporal dependencies between observations (section 2.2.2). We are given a set of explanatory variables $\boldsymbol{x}_{i}$ that encode experimental conditions or subgroups, e.g., element one is the intercept 1 and element two is 0 for conventional and 1 for short sleepers. We bind the $\boldsymbol{x}_{i}$ 's row-wise into the usual design matrix $\boldsymbol{X}$. Our model:

$$
\boldsymbol{y}_{i}=\gamma_{i} \times \boldsymbol{B} \boldsymbol{x}_{i}+\boldsymbol{\epsilon}_{i} \quad \text { for } \quad i=1, \ldots, n
$$

has a random effects term $\gamma_{i} \times \boldsymbol{B} \boldsymbol{x}_{i}$ and an independent and identically distributed error term $\epsilon_{i}$. We suppose the two random variables to have:

$$
\begin{array}{lll}
\mathrm{E}\left(\boldsymbol{\epsilon}_{i}\right)=0, & \operatorname{Cov}\left(\boldsymbol{\epsilon}_{i}\right)=\boldsymbol{\sigma}^{2} \boldsymbol{I}_{p} \\
\mathrm{E}\left(\gamma_{i}\right)=0, & \operatorname{Var}\left(\gamma_{i}\right)=1, & \mathrm{E}\left(\gamma_{i} \times \boldsymbol{\epsilon}_{i}\right)=0 .
\end{array}
$$

Then, the expected covariance is of the form:

$$
\mathrm{E}\left(\boldsymbol{y}_{i} \boldsymbol{y}_{i}^{T}\right)=\boldsymbol{B} \boldsymbol{x}_{i} \boldsymbol{x}_{i}^{T} \boldsymbol{B}^{T}+\boldsymbol{\sigma}^{2} \boldsymbol{I}_{p}=\boldsymbol{\Sigma}_{\boldsymbol{x}_{i}}
$$

resulting from the inclusion of the random variable $\gamma_{i}$. The covariance matrix $\boldsymbol{\Sigma}$ is indexed by $\boldsymbol{x}_{i}$ to indicate that it changes as a function of the explanatory variables. As with usual univariate linear modeling, we can interpret the coefficients $\boldsymbol{B}$ as explaining differences between experimental conditions. The matrix $\boldsymbol{B}$ is $p \times J$ dimensional, where $J$ is the number of columns in the $n \times J$ dimensional design matrix $\boldsymbol{X}$. Here $J=2$ and the second column encodes the contrast between short sleepers and conventional sleepers. The interpretation of $\boldsymbol{B}$ is that small values indicate little heteroscedasticity, identical signs indicates positive correlation, and opposite signs indicate negative correlation. For instance, assume that the second column of $\boldsymbol{B}$ is $\boldsymbol{b}_{2}=(-1,3,0,2)^{T}$. The interpretation for these four regions is as follows: region one and two are negatively correlated, so are region one and four, region two and four are positively correlated, and region three is uncorrelated.

The general form of this model was introduced by Hoff and Niu (2012) with the idea of decomposing covariance matrices into covariates explained and unexplained terms. In this original form the unexplained part is parametrized as a full covariance matrix scaling quadratically in the number of regions, i.e., $p(p-1) / p$ parameters. Instead, we parametrize it as a diagonal matrix with independent variance terms for each region. This simplified model scales linearly in the number of regions $p$ and can therefore be applied to large brain parcellations.

We use flat priors on both parameters $\sigma$ and $\boldsymbol{B}$. The elements of the $\boldsymbol{B}$ matrix have a uniform prior on $(-\infty, \infty)$, and the elements of $\sigma$ vector have a uniform prior on $(0, \infty)$. These priors are improper and do not integrate to one over their support. In case of prior knowledge, it is preferable to use more informative priors. For large $p$, we can add an additional hierarchical level to adjusting for multiple testing by including a common inclusion probability per column in $\boldsymbol{B}$ (Scott and Berger, 2006, 2010).

As is common in univariate linear modeling, it is possible to encode additional explanatory variables such as subject ID and possible batch factors. It would also be possible to extend the model to include temporal dependencies in the form of spline coefficients. We have not done so here because we wanted to focus explicitly on functional connectivity between regions.

\subsubsection{Effective Sample Size}

We subsample $n$ time points to obtain the Effective Sample Size (ESS). This $n$ is smaller than the total number $N$ of time points because it accounts for temporal dependency. We propose a procedure to automatically choose $n$ using an autocorrelation estimate of the timeseries. This is current practice in the field of Markov chain Monte Carlo and implemented in $\mathrm{R}$ package coda (Plummer et al., 2006). The ESS describes how much 
a dependent sample is worth with respect to an independent sample of the same size. Kass et al. (1998) define ESS via the lag $t$ autocorrelation $\operatorname{Corr}\left(\boldsymbol{y}_{1}^{(j)}, \boldsymbol{y}_{1+t}^{(j)}\right)$ as:

$$
n=\min _{j=1, \ldots, p}\left(\frac{N}{1+2 \sum_{t=1}^{\infty} \operatorname{Corr}\left(y_{1}^{(j)}, y_{1+t}^{(j)}\right)}\right) .
$$

This is a component-wise definition and we follow a conservative approach by taking the minimum over all $p$ components as the overall estimator. Intuitively, the larger the autocorrelation the lower is our ESS because we can predict future form current time points. A convenient side-produce of subsampling is reduced computational costs.

\subsubsection{Inference}

We implement our model in the probabilistic programming language Stan (Carpenter et al., 2017) using R. Stan uses Hamiltonian Monte Carlo to sample efficiently from posterior distributions using automatic differentiation. It removes the need for manually deriving gradients of the posterior distributions, thus making it easy to extend models. Our Stan code is available in our new R package CovRegFC from our GibHub repository. Alternatively, using conjugate priors it is possible to derive a Gibbs sampler to sample from the posterior distribution of a related model as in Hoff and Niu (2012). However, this makes it harder to extend the model.

Due to the non-identifiability of matrix $\boldsymbol{B}$ up to random sign changes, $\boldsymbol{B}$ and $-\boldsymbol{B}$ corresponding to the same covariance function, we need to align the posterior samples coming from multiple chains. A general option is to use Procrustes alignment. Procrustes alignment (Korth and Tucker, 1976) is a method for landmark registration (Kendall, 1984; Bookstein, 1986) in the shape statistics literature and an implementation is available in the R package shape (Dryden and Mardia, 1998).

\subsection{Full Covariance Regression}

In this section, we introduce a full covariance linear model.

\subsubsection{Model}

Here we do not subsample and deal with temporal dependencies in a different way. In this model, the number of observations are the number of subjects $k=1, \ldots, K$. After column-wise centering of each $N \times p$ (recall that $N$ is the total number of time points) timeseries $\boldsymbol{Y}_{1}, \ldots, \boldsymbol{Y}_{K}$, we compute sample covariance matrices for each subject $\boldsymbol{S}_{1}=\boldsymbol{Y}_{1}^{T} \boldsymbol{Y}_{1}, \ldots, \boldsymbol{S}_{K}=\boldsymbol{Y}_{K}^{T} \boldsymbol{Y}_{K}$. We take this as our "observed" response. Additionally, we have one explanatory vector $\boldsymbol{x}_{1}, \ldots, \boldsymbol{x}_{n}$ for each response covariance matrix. In our HCP data subset, we have 730 subjects, so $K=730$ and we have $K$ data point pairs $\left(\boldsymbol{S}_{1}, \boldsymbol{x}_{1}\right), \ldots,\left(\boldsymbol{S}_{K}, \boldsymbol{x}_{K}\right)$. We assume that the explanatory vector has two elements: the first element $x_{k}^{(1)}$ representing the intercept and is equal to one, and the second element $x_{k}^{(2)}$ is one for short and zero for conventional sleepers. Our regression model:

$$
\boldsymbol{S}_{k} \sim \text { Wishart }\left(x_{k}^{(1)} \boldsymbol{\Sigma}^{(1)}+x_{k}^{(2)} \boldsymbol{\Sigma}^{(2)}, v\right)
$$

decomposes the "observed" covariance matrix into an intercept term and a term encoding the functional connectivity between sleepers. The second parameter in the Wishart distribution describes the degrees of freedom and has support $(p-1, \infty)$.

We will now describe how to draw samples from the Wishart distribution, this will give us a better intuition for the proposed model. Matrices following a Wishart distribution can be generated by drawing vectors $y_{1}, \ldots, y_{N}$ independently from a $\operatorname{Normal}(0, \boldsymbol{\Sigma})$, storing vectors in a $N \times p$ matrices $\boldsymbol{Y}_{i}$, and computing the sample covariance matrix $\boldsymbol{S}_{i}=\boldsymbol{Y}_{i}^{T} \boldsymbol{Y}_{i}$. Then, the constructed $S_{i}$ 's are distributed according to a Wishart distribution with parameters $\boldsymbol{\Sigma}$ and degrees of freedom $N$. If the ESS is smaller than $N$ it will be reflected in the degrees of freedom parameter $v$. In our model, we will estimate $v$ from the data. In this way, we account for the temporal dependencies in the timeseries. The marginal posterior distribution of $v$ will be highly concentrated around a small degree of freedom (close to $p$ ) for strongly dependent samples and concentrated around a large degree of freedom (close to $N$ ) for weakly dependent samples.

To complete our model description, we need to put priors on covariance matrices and the degrees of freedom. We decompose the covariance prior into a standard deviation $\sigma$ vector and a correlation matrix $\boldsymbol{\Omega}$ for each term:

$$
\boldsymbol{\Sigma}^{(1)}=\boldsymbol{\sigma}^{(1)} \boldsymbol{I}_{p} \boldsymbol{\Omega}^{(1)} \boldsymbol{\sigma}^{(1)} \boldsymbol{I}_{p} \quad \text { and } \quad \boldsymbol{\Sigma}^{(2)}=\boldsymbol{\sigma}^{(2)} \boldsymbol{I}_{p} \boldsymbol{\Omega}^{(2)} \boldsymbol{\sigma}^{(2)} \boldsymbol{I}_{p}
$$

and put a Lewandowski, Kurowicka, and Joe (LKJ) prior on the correlation matrix (Lewandowski et al., 2009) independently for each term:

$$
\boldsymbol{\Omega}^{(1)} \sim \operatorname{LKJ} \operatorname{corr}(\eta) \quad \text { and } \quad \boldsymbol{\Omega}^{(2)} \sim \operatorname{LKJ} \operatorname{corr}(\eta) .
$$

This correlation matrix prior has one parameter $\eta$ that defines the amount of expected correlations. To gain intuition about $\eta$, we draw samples from the prior for a range of dimensions and parameter settings (Figure 2). The behavior in two dimension is similar to a beta distribution putting mass on either the boundary of the support of the prior or in the center. As we move toward higher dimensions, we can see that the distribution is less sensitive to the parameter $\eta$. For our model, we set $\eta=1$ to enforce a flat prior. We complete our prior description by putting independent flat priors on both the vector of standard deviations $\sigma$ and the degrees of freedom $v$, i.e., uniform prior on $(0, \infty)$ and uniform prior on $(p-1, N-1)$, respectively.

\subsubsection{Inference}

The number of parameters in the model scales quadratically in the number of regions making this model applicable in the classical statistical setting where we have larger sample sizes than number of predictors. In section 3.1, we will show an application to the HCP data with $K=730$ subjects and $p=15$ regions. Note, Hoff (2009) devised a Gibbs sampler for a similar model using an eigenmodel for the subject-level covariance matrices.

\subsubsection{Posterior Analysis and Multiplicity Control}

After drawing samples from the posterior, we can evaluate the marginal posterior distributions of standard deviations $\sigma$, correlations $\boldsymbol{\Omega}$, and degrees of freedom $\nu$. As mentioned, we 


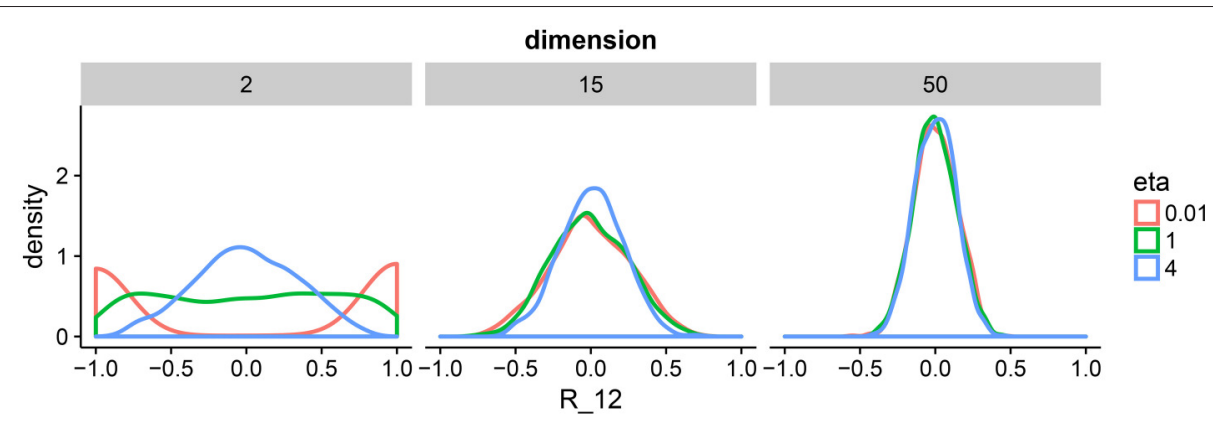

FIGURE 2 | Density plots of 1,000 draws from the LKJ prior in 2, 15, and 50 dimensions. We plot one off-diagonal element in the correlation matrix for each matrix $R$ drawn form the LKJ prior. We pick element $(1,2)$ denoted by $R_{12}$. This is representative of the other elements in the matrix because the LKJ prior is symmetric. We evaluate different shape parameters $\eta$. In the low-dimensional setting, a small $\eta$ assigns larger probability mass to correlations close to - 1 and 1 , whereas a large $\eta$ assigns larger mass around 0 . In the high-dimensional setting, probability mass is concentrated around 0 irrespectively of $\eta$. The special case of $\eta=1$ represents the uniform distribution on correlation matrices.

assume that the second element in the explanatory vector encodes whether a subject is a short or a conventional sleepers. In this setting, $\boldsymbol{\Omega}^{(2)}$ represents the difference in correlation between short and conventional sleepers. As we have the marginal posterior distribution for every $\Omega_{i j}^{(2)}$, we can evaluate the probability:

$$
P_{i j}=\left|2 \operatorname{Prob}\left(\Omega_{i j}^{(2)}>0\right)-1\right| .
$$

Our interpretation in terms of connectivity is as follows: If $P_{i j}$ is zero then the correlation is equally probable to be negative or positive. In this case, we are unable to clearly classify the sign of the correlation difference as negative or positive. If $P_{i j}$ is close to one then the correlation is more probable to be either negative or positive. In this case, we can say that parcel $i$ can be seen to be differentially connected to parcel $j$.

There are $p(p-1) / 2$ pairwise correlations and we wish to find correlations that are different between the two groups. If the probability $P_{i j}$ is large, we will report the connection as significantly different. To control for multiple testing, we declare correlations only as significant if they pass a threshold $\lambda$. We choose $\lambda$ to control the posterior expected FDR (Mitra et al., 2016):

$$
\mathrm{FDR}_{\lambda}=\frac{\sum_{i j}\left(1-P_{i j}\right) I\left(P_{i j}>\lambda\right)}{\sum_{i j} I\left(P_{i j}>\lambda\right)} .
$$

We find $\lambda$ through grid search for a fixed FDR. This allow us to report only correlations that survive the threshold at a given FDR.

\section{RESULTS}

The HCP released a dataset with 820 timeseries of normal healthy subjects measured during resting-state fMRI (rfMRI). The imaging data is accompanied by demographic and behavioral data including a sleep questionnaire. Approximately 30\% Americans are reported short sleepers with $4-6 \mathrm{~h}$ of sleep per night. The National Sleep Foundation recommends that adults sleep between 7 and $9 \mathrm{~h}$. We use both models to analyze the HCP data on 730 participants (after subsetting to short and conventional sleepers) to elucidate difference in functional connectivity between short and conventional sleepers. As mentioned before, the design matrix $\boldsymbol{X}$ has an intercept 1 and a column encoding short sleepers 1 and conventional sleepers 0 , i.e., conventional sleepers are the reference condition. We use a burn-in of 500 steps during which Stan optimizes tuning parameters for the HMC sampler, e.g., the mass matrix and the integration step length. After burn-in, we run HMC for additional 500 steps. To check convergence, we assess traceplots of random parameter subsets. We obtain an effective sample size of 167 for the 15 regions ICA-based parcellation. We now analyze the marginal posterior distribution of each of the parameters.

\subsection{Differential Analysis \\ 3.1.1. Fifteen Parcels}

In Figure 3, we summarize and visualize the marginal posterior distribution of the second column in $\boldsymbol{B}$. In the center part of the plot, we show the posterior distribution as posterior medians (dot) and credible intervals containing 95\% of the posterior density (segments). The credible intervals are Bonferroni corrected by fixing the segment endpoints at the $0.05 / 15$ and $(1-0.05 / 15)$ quantiles. Care has to be taken when interpreting the location of segments with respect to the zero coefficient line (red line). Due to the sign non-identifiability of $\boldsymbol{B}$, we have to ignore on which side the segments are located. Recall that regions on the same side are positively correlated, regions on opposite sides are negatively correlation, and regions overlapping the red line are undecided. To relate the region name back to the anatomy, we plotted the most relevant axial slice in the MNI152 space on the left and the right of the coefficient plot, depending on their sign, respectively. We can make the following observations: Parcels in set 1 (R4, R5, R7, and R9) are positively correlated. Keep in mind that the sign of the coefficient carries no information about the sign of the correlation. So, even though the coefficients are negative the correlations are positive, because they are on the same side of the red line. Parcels in set 2 (R1-R3, $\mathrm{R} 8, \mathrm{R} 10-\mathrm{R} 13$, and R15) are also positively correlated, for the same 


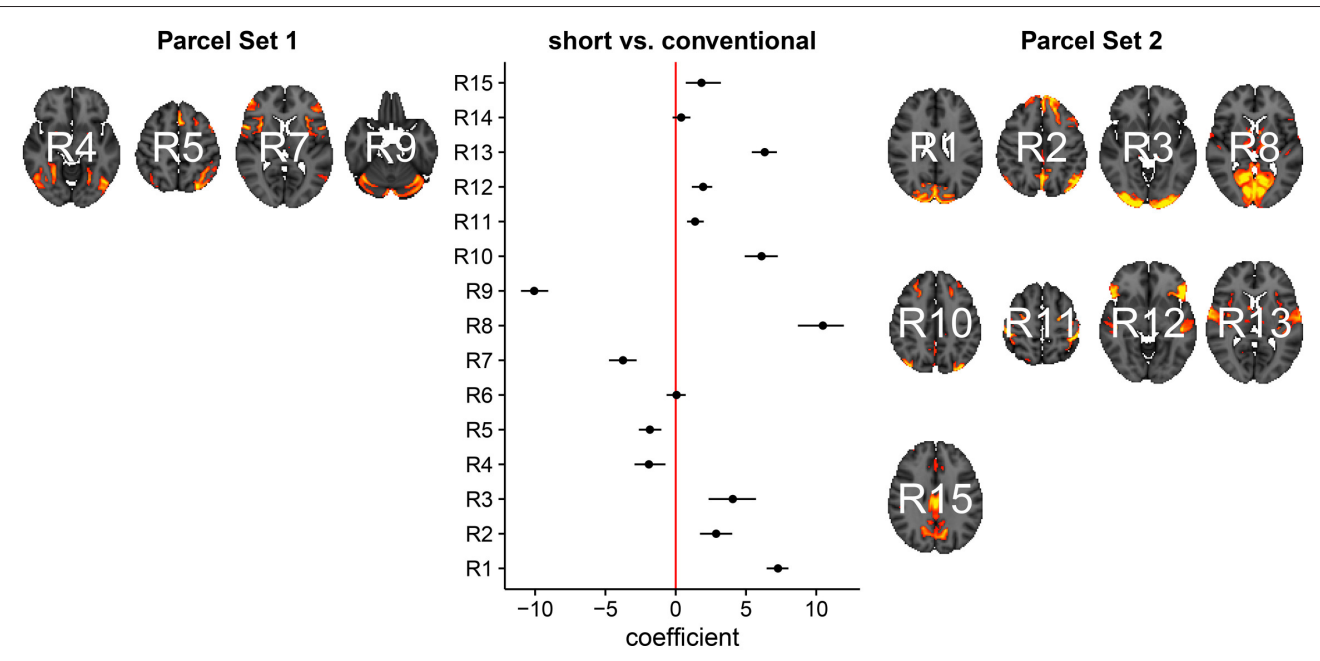

FIGURE 3 | Low-dimensional covariance model. This is the second column in the design matrix encoding the contrast between short and conventional sleepers. The sign is not identifiable; it only matters whether parcels are on the same or opposite side. If they are on the same side, then they are positively correlated. If they are on the opposite side, then they are negatively correlated. The posterior credible intervals are widened according to the number of regions or channels in the plot using the Bonferroni procedure.

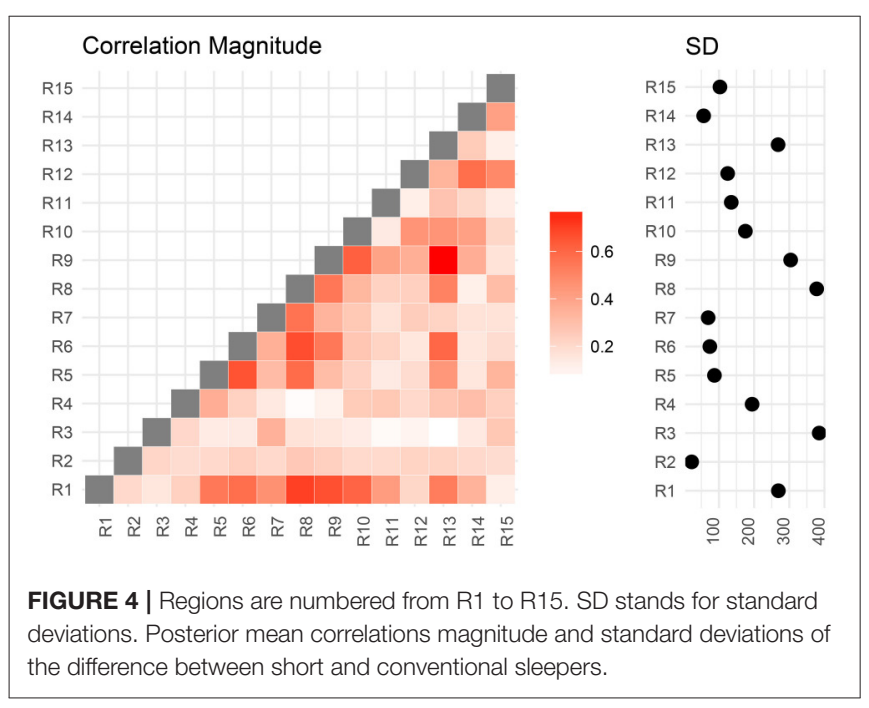

reason as before. In contrast, the two parcel sets are negatively correlated, because they are on opposite sides. The connectivity of R6 and R14 are not different from conventional sleepers because their credible intervals overlap the red line. According to the meta analysis in Smith et al. (2009), parcel set 1 is associate with visual, cognition-language, sensorimotor areas, and the cerebellum; and parcel set 2 with visual, cognition-language, auditory areas, and the default network.

We now compare the result from the low-dimensional model with results from the full model. First, we compute the posterior marginal mean of the standard deviations vector $\boldsymbol{\sigma}^{(2)}$ and the correlation matrix magnitude $\left|\boldsymbol{\Omega}^{(2)}\right|$ encoding the difference between short and conventional sleepers (Figure 4). The standard deviation plot on the right shows that parcel
$\mathrm{R} 3$ varies the most, and that region $\mathrm{R} 2$ varies the least. The magnitude correlation plot on the left shows that parcel pair R9 and R13 exhibit the strongest correlation. This is consistent with the low-dimensional model results, where R9 and R13 are in opposite parcel sets. Similarly, parcels R1 and R8 have a strong correlation magnitude in the full model and large effect sizes in the low-dimensional results.

In Figure 5, we assess the significance of differential correlations. The color code indicates different FDR levels. Overall strong differences in the correlation structure are visible with a large portion of connections at an FDR of 0.001. In contrast to the low-dimensional model, these are differences in correlations and not whether they are more positively or more negatively correlated.

\subsubsection{Fifty Parcels}

Modeling the data in a more compact representation makes it easier for us to interpret the results and easier to estimate parameters. For instance, consider analyzing $p=50$ parcels of 160 randomly sampled subjects form the HCP (Figure 6). All the information fits on one plot similar as in the $p=15$ parcel case. For $p=50$ it starts to get harder to interpret the full model because we have now 50(50-1)/2=1225 possible pairwise correlations. It will be hard to interpret a plot of the full correlation matrix. One way to make sense of it is to cluster rows and columns of the correlation matrix. Even though such postprocessing approaches are useful, it is unclear how to propagate uncertainty from the correlation estimation to the clustering step. A low-dimensional model is therefore our preferred analysis approach.

\subsubsection{Note on Computation Time}

For the low-dimensional model and the available 730 subjects, the computation time for the HMC sampler is around $20 \mathrm{~h}$ on 
a single core on a modern CPU. For a subsample of 40 subjects, the computation time is around $20-25 \mathrm{~min}$, and for 80 subjects around 50-55 min. It is possible to run more chains in parallel to increase the sample size. To combine each run, we need to align the posterior samples using Procrustes alignment as indicated in the section 2 .

The full model takes about $1 \mathrm{~h}$ on a single core, and we run four chains in parallel to increase sample size.

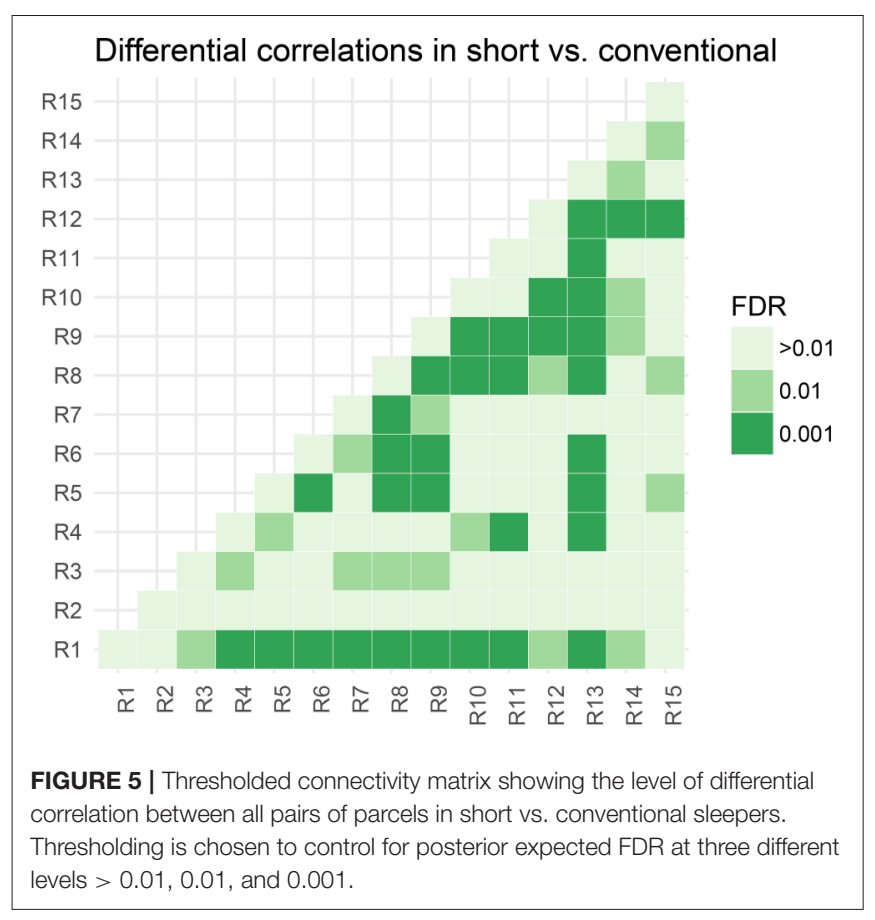

\subsection{Power Analysis}

We design a power analysis (Figure 7) for low-dimensional covariance regression with 15 parcels. As the population we take the available 730 subjects in the HCP data repository that are either short or conventional sleepers and have preprocessed timeseries. We sample 100 times from this population keeping the same ratio between the number of observations for each group, i.e., two thirds conventional and one third short sleepers. We report the average True Positive Rate (TPR) and the False Discovery Rate (FDR) over the 100 samples. We assign a parcel to a parcel set if its credible interval is located on one side of the zero red line and does not overlap the line. The credible intervals contain $100 \times(1-\alpha) \%$ of the marginal posterior distribution with end points evaluated using quantiles. We need to take into consideration that parcel sets are non-identifiable. We denote the $i$ th predicted parcel set as $Z_{i}^{\text {pred }}$ and the true parcel set as $Z_{i}^{\text {true }}$. The index $i$ can be either 1 or 2 . Parcel sets are subsets of $\{\mathrm{R} 1, \mathrm{R} 2, \ldots, \mathrm{R} 15\}$.

With these definitions, we are now ready to calculate TPR and FDR. The TPR measures the power of our procedure to detect true parcels. We define true positives as:

$$
\mathrm{TP}_{i j k l}=\#\left(Z_{i}^{\text {pred }} \cap Z_{j}^{\text {true }}\right)+\#\left(Z_{k}^{\text {pred }} \cap Z_{l}^{\text {true }}\right) .
$$

To obtain the rate, we take the maximum of both possible comparisons:

$$
\text { Correctly Predicted Parcels }=\max \left(\mathrm{TP}_{1122}, \mathrm{TP}_{1221}\right)
$$

and divide by the total number of true parcels:

$$
\mathrm{TPR}=\frac{\text { Correctly Predicted Parcels }}{\text { Total True Parcels }} .
$$

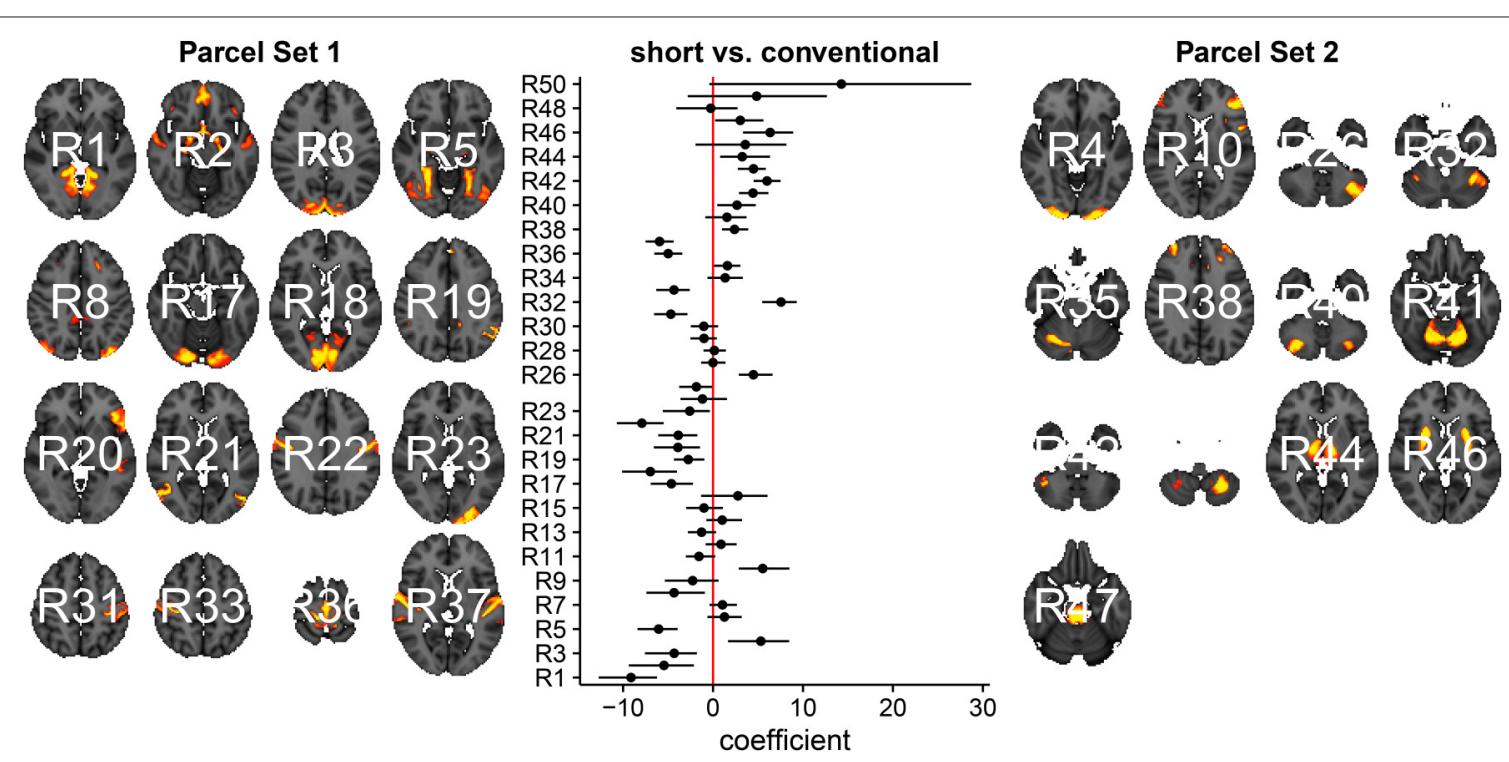

FIGURE 6 | Low-dimensional covariance model applied to 50 parcels of 160 randomly sampled subjects from the HCP after subsetting to short and conventional sleepers. 


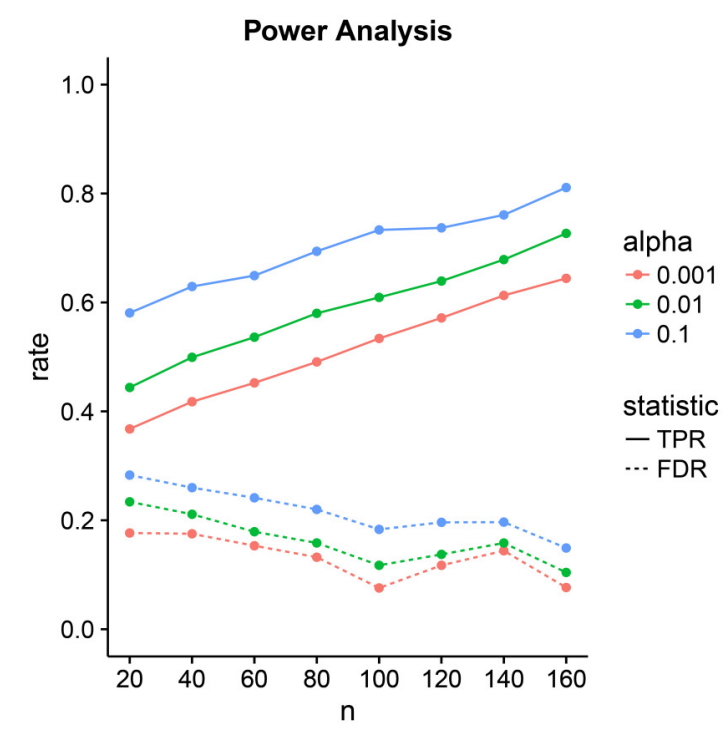

FIGURE 7 | Power analysis for low-dimensional covariance regression with 15 parcels. The two statistics are the True Positive Rate (TPR) and the False Discovery Rate (FDR). The significance level is denoted by $\alpha$. Points are averages computed over 100 samples from the population.

The FDR measures the amount of falsely predicted parcels as:

$$
\mathrm{FP}_{i j k l}=\#\left(Z_{i}^{\text {pred }} \backslash Z_{j}^{\text {true }}\right)+\#\left(Z_{k}^{\text {pred }} \backslash Z_{l}^{\text {true }}\right)
$$

by taking the minimum

$$
\text { Falsely Predicted Parcels }=\min \left(\mathrm{FP}_{1122}, \mathrm{FP}_{1221}\right)
$$

divided by the total number of positives

$$
\text { FDR }=\frac{\text { Falsely Predicted Parcels }}{\text { Correctly Predicted Parcels }+ \text { Falsely Predicted Parcels }} .
$$

The tradeoff between the two can be controlled through the significance level $\alpha$. Power increases linearly with sample size. FDR decrease linearly but at a lower rate with sample size. At samples size 40 , we have a power of $50 \%$ with an FDR of $20 \%$. This improves to a power of $80 \%$ with an FDR of $10 \%$ at sample size 160 .

\section{DISCUSSION}

We introduced two new models for functional connectivity. In particular, the low-dimensional covariance model is able to discover $50 \%$ of the correlation differences at a FDR of $20 \%$ in a sample size as little as 40 . Our Stan implementations make it easy for others to extend our models. We applied both models to the HCP data subset to compare functional connectivity between short and conventional sleepers. Our findings are consistent with Curtis et al. (2016) and Killgore et al. (2012) reporting increases in functional connectivity in short sleepers for primary auditory, primary motor, primary somatosensory, and primary visual cortices. A similar neural signature was observed in experiments examining the transition from resting wakefulness to sleep onset using EEG and rfMRI (Larson-Prior et al., 2009; Tagliazucchi and Laufs, 2014; Davis et al., 2016). Therefore, we recommend the inclusion of the average sleep duration of a subject as a "batch" covariate in the experimental design of rfMRI studies.

In addition to group comparisons encoded as a design matrix with two columns, it is possible to extend our low-dimensional model to more complicated experimental designs by appending more columns to the design matrix. We can encode batch factors and subject-specific variability by binding one column per factor level. Besides categorical variables, we can model continuous variables such as head-motion measurement made using an accelerometer. Adding covariates to explain unwanted variation in the data can move some of the preprocessing steps to the functional connectivity analysis step. Such joint modeling can enable the propagation of uncertainty to the downstream analyses. Additional columns in the design matrix are called blocking factors and can improve the statistical power. Without modeling the blocking factor, the variability in the data is absorbed by the noise term. The higher level of noise leads to higher uncertainty in our parameter estimates. In contrast, a model with additional blocking factors has more parameters that need to be estimated. As in most practical problems, the right modeling choice depends on the data.

A main challenge in covariance regression is the positive definiteness constraint. A solution is to transform the covariance estimation problem into an unconstrained problem thus making estimation and inference easier (Pourahmadi, 2011). One possible transformation starts with a spectral decomposition where the covariance matrix is decomposed into a diagonal matrix of eigenvalues and an orthogonal matrix with normalized eigenvectors as columns. The procedure continues with a global log-transformation to the covariance matrix, which results in a log-transformation of individual eigenvalues and removes the constraint. However, mathematically and computationally tempting this approach seems, it remains hard to interpret the log-transformations statistically (Brown et al., 1994; Liechty et al., 2004). An alternative transformation uses a Cholesky decomposition of the covariance matrix. For the Cholesky decomposition, we need a natural ordering of the variables not known a priori for functional connectivity data-a natural ordering could be given if the chronology is known.

Modeling of covariance matrices builds on important geometrical concepts and the medical image analysis community has made significant progress in terms of mathematical descriptions and practical applications motivated by data in diffusion tensor imaging (Pennec, 1999, 2006; Moakher, 2005; Arsigny et al., 2006/2007; Lenglet et al., 2006; Fletcher and Joshi, 2007; Fillard et al., 2007; Schwartzman et al., 2008; Dryden et al., 2009). The underlying geometry is called Lie group theory and it appears when we consider the covariance matrices as elements in a non-linear space. The matrix log-transformation from the previous paragraph maps covariance matrices to the tangent space where unconstrained operations can be performed; for instance we create a mean by simple elementwise averaging. After computing the mean in tangent space, this mean is mapped 
back to the constrained space of covariance matrices. Despite the mathematical beauty and algorithmic convenience, statistical interpretations are still unwieldy. However, this does provide a fundamental geometric formulation and enables the use of handy geometrical tools (Absil et al., 2008 for a book-length treatment).

We approach the problem from a statistical viewpoint and frame functional connectivity in terms of modeling heteroscedasticity. This allows us to take advantage of the rich history in statistics and led us to the covariance regression model introduced by Hoff and Niu (2012). We simplify the model to meet the large $p$ requirement in neuroscience. The running time for 500 posterior samples on 80 subjects is less than an hour on a single core. This makes our approach applicable to many neuroimaging studies. For larger studies, such as the HCP with 730 subjects, further speed improvements using GPU's are desirable to reduce computation time.

One possible future application is functional Near-Infrared Spectroscopy (fNIRS), which has gained in popularity due its portability and high temporal resolution. A common approach is to set up a linear model between brain responses at channels locations (Huppert et al., 2009; Ye et al., 2009; Tak and Ye, 2014) and experimental conditions. Thus, our models apply to fNIRS experiments. An additional challenge in fNIRS experiments is channel registration across multiple participants (Liu et al., 2016). Connectivity differences could be due artifacts created by channel misalignments not biology. In the absence of structural MRI, we could add an additional hierarchical level in our low-dimensional model to handle measurement errors accounting for possible misalignments between channels.

We use a conservative component-wise estimate of the ESS. Less conservative multivariate estimators (Vats et al., 2015) might be able to increase statistical power at the cost of an increase in the false discovery rate.

\section{REPRODUCIBILITY AND SUPPLEMENTARY MATERIAL}

The entire data analysis workflow is available on our GitHub repository:

\section{REFERENCES}

Absil, P.-A., Mahony, R., and Sepulchre, R. (2008). Optimization Algorithms on Matrix Manifolds. Princeton, NJ: Princeton University Press .

Achard, S., Salvador, R., Whitcher, B., Suckling, J., and Bullmore, E. (2006). A resilient, low-frequency, small-world human brain functional network with highly connected association cortical hubs. J. Neurosci. 26, 63-72. doi: 10.1523/JNEUROSCI.3874-05.2006

Anderson, T. (1973). Asymptotically efficient estimation of covariance matrices with linear structure. Ann. Stat. 1, 135-141. doi: 10.1214/aos/11933 42389

Arsigny, V., Fillard, P., Pennec, X., and Ayache, N. (2006/2007). Geometric means in a novel vector space structure on symmetric positive-definite matrices. SIAM J. Matrix Anal. Appl. 29, 328-347. doi: 10.1137/0506 37996

Bassett, D. S., and Bullmore, E. (2006). Small-world brain networks. Neuroscientist 12, 512-523. doi: $10.1177 / 1073858406293182$
- https://github.com/ChristofSeiler/CovRegFC_HCP

We also provide a new R package CovRegFC with Stan code:

- https://github.com/ChristofSeiler/CovRegFC

Data preparation and statistical analyses are contained in Rmd files:

- Low_Dimensional.Rmd

- Full.Rmd

- Power. Rmd

By running these files all results and plots can be completely reproduced as html files:

- Low_Dimensional.html

- Full.html

- Power.html

The HCP data is available here:

- https://www.humanconnectome.org/data/

\section{AUTHOR CONTRIBUTIONS}

CS wrote an initial draft, performed and implemented the statistical analysis. SH wrote the final manuscript and provided statistical tools.

\section{ACKNOWLEDGMENTS}

CS was partially funded by two Swiss NSF postdoctoral fellowships 146281 and 158500 . SH was partially supported by NSF DMS grant 1501767. We thank the NIRS lab at the Center for Interdisciplinary Brain Sciences in the Stanford School of Medicine for introducing us to functional neuroimaging data in the context of fNIRS experiments.

Data were provided by the Human Connectome Project, WUMinn Consortium (Principal Investigators: David Van Essen and Kamil Ugurbil; 1U54MH091657) funded by the $16 \mathrm{NIH}$ Institutes and Centers that support the NIH Blueprint for Neuroscience Research; and by the McDonnell Center for Systems Neuroscience at Washington University.

Beckmann, C. F., and Smith, S. M. (2004). Probabilistic independent component analysis for functional magnetic resonance imaging. IEEE Trans. Med. Imaging 23, 137-152. doi: 10.1109/TMI.2003.822821

Biswal, B. B., Mennes, M., Zuo, X.-N., Gohel, S., Kelly, C., Smith, S. M., et al. (2010). Toward discovery science of human brain function. Proc. Natl. Acad. Sci. U.S.A. 107, 4734-4739. doi: 10.1073/pnas.0911855107

Bookstein, F. L. (1986). Size and shape spaces for landmark data in two dimensions. Stat. Sci. 1, 181-222. doi: 10.1214/ss/1177013696

Brown, P. J., Le, N. D., and Zidek, J. V. (1994). "Inference for a covariance matrix," in Aspects of Uncertainty: A Tribute to DV Lindley, eds P. Freeman and A. Smith (Chichester: Wiley), 77-92.

Bullmore, E., and Sporns, O. (2009). Complex brain networks: graph theoretical analysis of structural and functional systems. Nat. Rev. Neurosci. 10, 186-198. doi: 10.1038/nrn2575

Carpenter, B., Gelman, A., Hoffman, M., Lee, D., Goodrich, B., Betancourt, M., et al. (2017). Stan: a probabilistic programming language. J. Stat. Soft. 76, 1-32. doi: $10.18637 /$ jss.v076.i01 
Craddock, R. C., James, G. A., Holtzheimer, P. E., Hu, X. P., and Mayberg, H. S. (2012). A whole brain fmri atlas generated via spatially constrained spectral clustering. Hum. Brain Mapp. 33, 1914-1928. doi: 10.1002/hbm.21333

Curtis, B. J., Williams, P. G., Jones, C. R., and Anderson, J. S. (2016). Sleep duration and resting fmri functional connectivity: examination of short sleepers with and without perceived daytime dysfunction. Brain Behav. 6:e00576. doi: 10.1002/brb3.576

Davis, B., Tagliazucchi, E., Jovicich, J., Laufs, H., and Hasson, U. (2016). Progression to deep sleep is characterized by changes to bold dynamics in sensory cortices. Neuroimage 130, 293-305. doi: 10.1016/j.neuroimage. 2015.12.034

Dosenbach, N. U., Nardos, B., Cohen, A. L., Fair, D. A., Power, J. D., Church, J. A., et al. (2010). Prediction of individual brain maturity using fMRI. Science 329, 1358-1361. doi: 10.1126/science.1194144

Dryden, I. L., Koloydenko, A., and Zhou, D. (2009). Non-Euclidean statistics for covariance matrices, with applications to diffusion tensor imaging. Ann. Appl. Stat. 3, 1102-1123. doi: 10.1214/09-AOAS249

Dryden, I. L., and Mardia, K. V. (1998). Statistical Shape Analysis. Wiley Series in Probability and Statistics: Probability and Statistics. Chichester: John Wiley \& Sons, Ltd.

Eguíluz, V. M., Chialvo, D. R., Cecchi, G. A., Baliki, M., and Apkarian, A. V. (2005). Scale-free brain functional networks. Phys. Rev. Lett. 94:018102. doi: 10.1103/PhysRevLett.94.018102

Fillard, P., Pennec, X., Arsigny, V., and Ayache, N. (2007). Clinical dt-mri estimation, smoothing, and fiber tracking with log-Euclidean metrics. IEEE Trans. Med. Imaging 26, 1472-1482. doi: 10.1109/TMI.2007.899173

Fischl, B., Sereno, M. I., and Dale, A. M. (1999). Cortical surface-based analysis: Ii: inflation, flattening, and a surface-based coordinate system. Neuroimage 9, 195-207. doi: 10.1006/nimg.1998.0396

Fletcher, P. T., and Joshi, S. (2007). Riemannian geometry for the statistical analysis of diffusion tensor data. Signal Process. 87, 250-262. doi: 10.1016/j.sigpro.2005.12.018

Fox, M. D., and Greicius, M. (2010). Clinical applications of resting state functional connectivity. Front. Syst. Neurosci. 4:19. doi: 10.3389/fnsys.2010.00019

Fox, M. D., and Raichle, M. E. (2007). Spontaneous fluctuations in brain activity observed with functional magnetic resonance imaging. Nat. Rev. Neurosci. 8, 700-711. doi: 10.1038/nrn2201

Friedman, J., Hastie, T., and Tibshirani, R. (2008). Sparse inverse covariance estimation with the graphical lasso. Biostatistics 9, 432-441. doi: 10.1093/biostatistics/kxm045

Friston, K. J. (2011). Functional and effective connectivity: a review. Brain Connect. 1, 13-36. doi: 10.1089/brain.2011.0008

Ginestet, C. E., Li, J., Balachandran, P., Rosenberg, S., and Kolaczyk, E. D. (2017). Hypothesis testing for network data in functional neuroimaging. Ann. Appl. Stat. 11, 725-750. doi: 10.1214/16-AOAS1015

Glasser, M. F., Sotiropoulos, S. N., Wilson, J. A., Coalson, T. S., Fischl, B., Andersson, J. L., et al. (2013). The minimal preprocessing pipelines for the human connectome project. Neuroimage 80, 105-124. doi: 10.1016/ j.neuroimage.2013.04.127

Greicius, M. (2008). Resting-state functional connectivity in neuropsychiatric disorders. Curr. opin. Neurol. 21 424-430. doi: 10.1097/WCO. 0b013e328306f2c5

Greicius, M. D., Krasnow, B., Reiss, A. L., and Menon, V. (2003). Functional connectivity in the resting brain: a network analysis of the default mode hypothesis. Proc. Natl. Acad. Sci. U.S.A. 100, 253-258. doi: $10.1073 /$ pnas. 0135058100

Griffanti, L., Salimi-Khorshidi, G., Beckmann, C. F., Auerbach, E. J., Douaud, G., Sexton, et al. (2014). ICA-based artefact removal and accelerated fmri acquisition for improved resting state network imaging. Neuroimage 95, 232-247. doi: 10.1016/j.neuroimage.2014.03.034

Grillon, M.-L., Oppenheim, C., Varoquaux, G., Charbonneau, F., Devauchelle, A.-D., Krebs, M.-O., et al. (2013). Hyperfrontality and hypoconnectivity during refreshing in schizophrenia. Psychiatry Res. 211, 226-233. doi: 10.1016/j.pscychresns.2012.09.001

Hirshkowitz, M., Whiton, K., Albert, S. M., Alessi, C., Bruni, O., DonCarlos, L., et al. (2015). National sleep foundation's sleep time duration recommendations: methodology and results summary. Sleep Health 1, 40-43. doi: 10.1016/j.sleh.2014.12.010
Hoff, P. D. (2009). A hierarchical eigenmodel for pooled covariance estimation. J. R. Stat. Soc. Ser. B Stat. Methodol. 71, 971-992. doi: 10.1111/j.1467-9868.2009.00716.x

Hoff, P. D., and Niu, X. (2012). A covariance regression model. Statist. Sinica 22, 729-753. doi: $10.5705 /$ ss.2010.051

Huppert, T. J., Diamond, S. G., Franceschini, M. A., and Boas, D. A. (2009). Homer: a review of time-series analysis methods for near-infrared spectroscopy of the brain. Appl. Opt. 48, D280-D298. doi: 10.1364/AO.48.00D280

Hyvärinen, A. (1999). Fast and robust fixed-point algorithms for independent component analysis. IEEE Trans. Neural Netw. 10, 626-634. doi: 10.1109/72.761722

Jenkinson, M., Beckmann, C. F., Behrens, T. E., Woolrich, M. W., and Smith, S. M. (2012). FSL. Neuroimage 62, 782-790. doi: 10.1016/j.neuroimage.2011.09.015

Kass, R. E., Carlin, B. P., Gelman, A., and Neal, R. M. (1998). Markov chain monte carlo in practice: a roundtable discussion. Am. Stat. 52, 93-100. doi: $10.2307 / 2685466$

Kendall, D. G. (1984). Shape manifolds, Procrustean metrics, and complex projective spaces. Bull. London Math. Soc. 16 81-121. doi: 10.1112/blms/16.2.81

Killgore, W. D., Schwab, Z. J., and Weiner, M. R. (2012). Self-reported nocturnal sleep duration is associated with next-day resting state functional connectivity. Neuroreport 23, 741-745. doi: 10.1097/WNR.0b013e3283565056

Korth, B., and Tucker, L. R. (1976). Procrustes matching by congruence coefficients. Psychometrika 41, 531-535. doi: 10.1007/BF02296973

Larson-Prior, L. J., Zempel, J. M., Nolan, T. S., Prior, F. W., Snyder, A. Z., and Raichle, M. E. (2009). Cortical network functional connectivity in the descent to sleep. Proc. Natl. Acad. Sci. U.S.A. 106, 4489-4494. doi: 10.1073/pnas.0900924106

Ledoit, O., and Wolf, M. (2004). A well-conditioned estimator for largedimensional covariance matrices. J. Multiv. Anal. 88, 365-411. doi: 10.1016/ S0047-259X(03)00096-4

Lenglet, C., Rousson, M., and Deriche, R. (2006). DTI segmentation by statistical surface evolution. IEEE Trans. Med. Imaging 25, 685-700. doi: 10.1109/TMI.2006.873299

Lewandowski, D., Kurowicka, D., and Joe, H. (2009). Generating random correlation matrices based on vines and extended onion method. J. Multiv. Anal. 100, 1989-2001. doi: 10.1016/j.jmva.2009.04.008

Lewis, C. M., Baldassarre, A., Committeri, G., Romani, G. L., and Corbetta, M. (2009). Learning sculpts the spontaneous activity of the resting human brain Proc. Natl. Acad. Sci. U.S.A. 106, 17558-17563. doi: 10.1073/pnas.0902455106

Liang, X., Connelly, A., and Calamante, F. (2016). A novel joint sparse partial correlation method for estimating group functional networks. Hum. Brain Mapp. 37, 1162-1177. doi: 10.1002/hbm.23092

Liechty, J. C., Liechty, M. W., and Müller, P. (2004). Bayesian correlation estimation. Biometrika 91, 1-14. doi: 10.1093/biomet/91.1.1

Liu, N., Mok, C., Witt, E. E., Pradhan, A. H., Chen, J. E., and Reiss, A. L. (2016). NIRS-based hyperscanning reveals inter-brain neural synchronization during cooperative Jenga game with face-to-face communication. Front. Hum. Neurosci. 10:82. doi: 10.3389/fnhum.2016.00082

Marrelec, G., Bellec, P., Krainik, A., Duffau, H., Pélégrini-Issac, M., Lehéricy, S., et al. (2008). Regions, systems, and the brain: hierarchical measures of functional integration in fmri. Med. Image Anal. 12, 484-496. doi: 10.1016/j.media.2008.02.002

Mitra, R., Müller, P., and Ji, Y. (2016). Bayesian graphical models for differential pathways. Bayesian Anal. 11, 99-124. doi: 10.1214/14-BA931

Moakher, M. (2005). A differential geometric approach to the geometric mean of symmetric positive-definite matrices. SIAM J. Matrix Anal. Appl. 26, 735-747. doi: $10.1137 /$ S0895479803436937

Pennec, X. (1999). "Probabilities and statistics on Riemannian manifolds: Basic tools for geometric measurements," in NSIP, eds A. Enis Cetin, L. Akarun, A. Ertüzün, M. N. Gurcan, and Y. Yardimci (Bogaziçi University Printhouse), 194-198.

Pennec, X. (2006). Intrinsic statistics on Riemannian manifolds: basic tools for geometric measurements. J. Math. Imaging Vis. 25, 127-154. doi: 10.1007/s10851-006-6228-4

Plummer, M., Best, N., Cowles, K., and Vines, K. (2006). CODA: Convergence diagnosis and output analysis for MCMC. R. News 6, 7-11.

Pourahmadi, M. (2011). Covariance estimation: the GLM and regularization perspectives. Stat. Sci. 26, 369-387. doi: 10.1214/11-STS358 
Preti, M. G., Bolton, T. A., and Ville, D. V. D. (2016). The dynamic functional connectome: state-of-the-art and perspectives. Neuroimage 160, 41-54. doi: 10.1016/j.neuroimage.2016.12.061

Ryali, S., Chen, T., Supekar, K., and Menon, V. (2012). Estimation of functional connectivity in fMRI data using stability selection-based sparse partial correlation with elastic net penalty. Neuroimage 59, 3852-3861. doi: 10.1016/j.neuroimage.2011.11.054

Salimi-Khorshidi, G., Douaud, G., Beckmann, C. F., Glasser, M. F., Griffanti, L., and Smith, S. M. (2014). Automatic denoising of functional MRI data: combining independent component analysis and hierarchical fusion of classifiers. Neuroimage 90, 449-468. doi: 10.1016/j.neuroimage.2013.11.046

Salvador, R., Suckling, J., Coleman, M. R., Pickard, J. D., Menon, D., and Bullmore, E. (2005). Neurophysiological architecture of functional magnetic resonance images of human brain. Cereb. Cortex 15, 1332-1342. doi: 10.1093/cercor/bhi016

Schwartzman, A., Dougherty, R. F., and Taylor, J. E. (2008). False discovery rate analysis of brain diffusion direction maps. Ann. Appl. Stat. 2, 153-175. doi: 10.1214/07-AOAS133

Scott, J. G., and Berger, J. O. (2006). An exploration of aspects of Bayesian multiple testing. J. Stat. Plann. Infer. 136, 2144-2162. doi: 10.1016/j.jspi.2005.08.031

Scott, J. G., and Berger, J. O. (2010). Bayes and empirical-Bayes multiplicity adjustment in the variable-selection problem. Ann Stat. 38, 2587-2619. doi: 10.1214/10-AOS792

Smith, S. M. (2012). The future of fMRI connectivity. Neuroimage 62, 1257-1266. doi: 10.1016/j.neuroimage.2012.01.022

Smith, S. M., Beckmann, C. F., Andersson, J., Auerbach, E. J., Bijsterbosch, J., Douaud, G., et al. (2013). Resting-state fMRI in the human connectome project. Neuroimage 80, 144-168. doi: 10.1016/j.neuroimage.2013.05.039

Smith, S. M., Fox, P. T., Miller, K. L., Glahn, D. C., Fox, P. M., Mackay, C. E., et al. (2009). Correspondence of the brain's functional architecture during activation and rest. Proc. Natl. Acad. Sci. U.S.A. 106, 13040-13045. doi: 10.1073/pnas.0905267106

Smith, S. M., Hyvärinen, A., Varoquaux, G., Miller, K. L., and Beckmann, C. F. (2014). Group-PCA for very large fMRI datasets. Neuroimage 101, 738-749. doi: 10.1016/j.neuroimage.2014.07.051

Smith, S. M., Miller, K. L., Salimi-Khorshidi, G., Webster, M., Beckmann, C. F., Nichols, T. E., et al. (2011). Network modelling methods for fMRI. Neuroimage 54, 875-891. doi: 10.1016/j.neuroimage.2010.08.063

Sporns, O., Chialvo, D. R., Kaiser, M., and Hilgetag, C. C. (2004). Organization, development and function of complex brain networks. Trends Cogn. Sci. 8, 418-425. doi: 10.1016/j.tics.2004.07.008

Stam, C. J. (2004). Functional connectivity patterns of human magnetoencephalographic recordings: a small-worldnetwork? Neurosci. Lett. 355, 25-28. doi: 10.1016/j.neulet.2003.10.063
Tagliazucchi, E., and Laufs, H. (2014). Decoding wakefulness levels from typical fmri resting-state data reveals reliable drifts between wakefulness and sleep. Neuron 82, 695-708. doi: 10.1016/j.neuron.2014.03.020

Tak, S., and Ye, J. C. (2014). Statistical analysis of fNIRS data: a comprehensive review. Neuroimage 85, 72-91. doi: 10.1016/j.neuroimage.2013.06.016

Van Den Heuvel, M. P., and Pol, H. E. H. (2010). Exploring the brain network: a review on resting-state fMRI functional connectivity. Eur. Neuropsychopharmacol. 20, 519-534. doi: 10.1016/j.euroneuro.2010. 03.008

Van Essen, D. C., Smith, S. M., Barch, D. M., Behrens, T. E., Yacoub, E., Ugurbil, K., et al. (2013). The WU-Minn human connectome project: an overview. Neuroimage 80, 62-79. doi: 10.1016/j.neuroimage.2013.05.041

Varoquaux, G., Baronnet, F., Kleinschmidt, A., Fillard, P., and Thirion, B. (2010a). "Detection of brain functional-connectivity difference in post-stroke patients using group-level covariance modeling," in International Conference on Medical Image Computing and Computer-Assisted Intervention (Beijing: Springer), 200-208.

Varoquaux, G., and Craddock, R. C. (2013). Learning and comparing functional connectomes across subjects. Neuroimage 80, 405-415. doi: 10.1016/j.neuroimage.2013.04.007

Varoquaux, G., Gramfort, A., Poline, J.-B., and Thirion, B. (2010b). "Brain covariance selection: better individual functional connectivity models using population prior," in Advances in Neural Information Processing Systems (Vancouver), 2334-2342.

Varoquaux, G., Gramfort, A., Poline, J. B., and Thirion, B. (2012). Markov models for fMRI correlation structure: is brain functional connectivity small world, or decomposable into networks? J. Physiol. 106, 212-221. doi: $10.1016 /$ j.jphysparis.2012.01.001

Vats, D., Flegal, J. M., and Jones, G. L. (2015). Multivariate output analysis for Markov chain Monte Carlo. arXiv preprint arXiv:1512.07713.

Ye, J. C., Tak, S., Jang, K. E., Jung, J., and Jang, J. (2009). NIRS-SPM: statistical parametric mapping for near-infrared spectroscopy. Neuroimage 44, 428-447. doi: 10.1016/j.neuroimage.2008.08.036

Conflict of Interest Statement: The authors declare that the research was conducted in the absence of any commercial or financial relationships that could be construed as a potential conflict of interest.

Copyright $\odot 2017$ Seiler and Holmes. This is an open-access article distributed under the terms of the Creative Commons Attribution License (CC BY). The use, distribution or reproduction in other forums is permitted, provided the original author(s) or licensor are credited and that the original publication in this journal is cited, in accordance with accepted academic practice. No use, distribution or reproduction is permitted which does not comply with these terms. 